J. Korean Math. Soc. 49 (2012), No. 4, pp. 687-701

http://dx.doi.org/10.4134/JKMS.2012.49.4.687

\title{
SPECIAL WEAK PROPERTIES OF GENERALIZED POWER SERIES RINGS
}

\author{
LUNQUN OUYANG
}

\begin{abstract}
Let $R$ be a ring and $\operatorname{nil}(R)$ the set of all nilpotent elements of $R$. For a subset $X$ of a ring $R$, we define $N_{R}(X)=\{a \in R \mid x a \in \operatorname{nil}(R)$ for all $x \in X\}$, which is called a weak annihilator of $X$ in $R$. A ring $R$ is called weak zip provided that for any subset $X$ of $R$, if $N_{R}(X) \subseteq \operatorname{nil}(R)$, then there exists a finite subset $Y \subseteq X$ such that $N_{R}(Y) \subseteq n i l(R)$, and a ring $R$ is called weak symmetric if $a b c \in \operatorname{nil}(R) \Rightarrow a c b \in \operatorname{nil}(R)$ for all $a, b, c \in R$. It is shown that a generalized power series $\operatorname{ring}\left[\left[R^{S, \leq}\right]\right]$ is weak zip (resp. weak symmetric) if and only if $R$ is weak zip (resp. weak symmetric) under some additional conditions. Also we describe all weak associated primes of the generalized power series ring $\left[\left[R^{S,} \leq\right]\right]$ in terms of all weak associated primes of $R$ in a very straightforward way.
\end{abstract}

\section{Introduction}

All rings considered here are associative with identity. Any concept and notation not defined here can be founded in Ribenboim [17-19], Elliott and Ribenboim [6], and L. Ouyang [15-16].

Let $(S, \leq)$ be an ordered set. Recall that $(S, \leq)$ is Artinian if every strictly decreasing sequence of elements of $S$ is finite, and that $(S, \leq)$ is narrow if every subset of pairwise order-incomparable elements of $S$ is finite. Let $S$ be a commutative monoid. Unless stated otherwise, the operation of $S$ shall be denoted additively, and the neutral element by 0 . The following definition is due to Elliott and Ribenboim [6].

Let $(S, \leq)$ be a strictly ordered monoid (that is, $(S, \leq)$ is an ordered monoid satisfying the condition that, if $s, s^{\prime}, t \in S$ and $s<s^{\prime}$, then $s+t<s^{\prime}+t$ ), and $R$ a ring. Let $\left[\left[R^{S, \leq}\right]\right]$ be the set of all maps $f: S \longrightarrow R$ such that $\operatorname{supp}(f)=\{s \in S \mid f(s) \neq 0\}$ is Artinian and narrow. With pointwise addition,

Received November 8, 2010; Revised May 22, 2011.

2010 Mathematics Subject Classification. 16W60, 16S36.

Key words and phrases. weak annihilator, weak associated prime, generalized power series.

This research is supported by the National Natural Science Foundation of China (11071062), Natural Science Foundation of Hunan Province (10jj3065) and Scientific Research Foundation of Hunan Provincial Education Department (10A033), Degree and Postgraduate Teaching Reform Foundation of Hunan Province (JG2009A017). 
$\left[\left[R^{S, \leq}\right]\right]$ is an abelian additive group. For every $s \in S$ and $f, g \in\left[\left[R^{S, \leq}\right]\right]$, let $X_{s}(f, g)=\{(u, v) \in S \times S \mid u+v=s, f(u) \neq 0, g(v) \neq 0\}$. It follows from [18, Section 4.1] that $X_{s}(f, g)$ is finite. This fact allows to define the operation of convolution:

$$
(f g)(s)=\sum_{(u, v) \in X_{s}(f, g)} f(u) g(v) .
$$

With this operation of convolution, and pointwise addition, $\left[\left[R^{S, \leq}\right]\right]$ becomes a ring (see [11-13] or [17-19]), which is called the generalized power series ring. The elements of $\left[\left[R^{S, \leq}\right]\right]$ are called generalized power series with coefficients in $R$ and exponents in $S$.

For example, let $\mathbb{N}$ denote the set of positive integers. If $S=\mathbb{N} \cup\{0\}$ and $\leq$

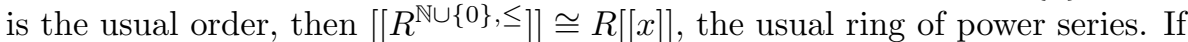
$S$ is a commutative monoid and $\leq$ is the trivial order, then $\left[\left[R^{S, \leq}\right]\right] \cong R[S]$, the monoid-ring of $S$ over $R$. Let $(S, \leq)$ be a strictly totally ordered monoid, which is also Artinian. For any $s \in S$, set $X_{s}=\{(u, v) \mid u+v=s, u, v \in S\}$. Then from [18, Section 4.1], it follows that $X_{s}$ is a finite set. Let $V$ be a free abelian additive group with the base consisting of elements of $S$. Then $V$ is a coalgebra over $\mathbb{Z}$ with the comultiplication map and the counit map as following:

$$
\Delta(s)=\sum_{(u, v) \in X_{s}} u \otimes v, \quad \varepsilon(s)= \begin{cases}1, & s=0 \\ 0, & s \neq 0 .\end{cases}
$$

Clearly $\left[\left[R^{S, \leq}\right]\right] \cong \operatorname{Hom}(V, R)$-the dual algebra.

Further examples and some properties of $\left[\left[R^{S, \leq}\right]\right]$ are given in [11-13] and [17-19].

Let $s \in S, r \in R$. We define $C_{r}^{s} \in\left[\left[R^{S,} \leq\right]\right]$ as follows:

$$
C_{r}^{s}(s)=r, \quad C_{r}^{s}(t)=0 \quad(s \neq t \in S) .
$$

Let $\left[\left[R^{S,} \leq\right]\right]$ be the generalized power series ring over $R$. Then $R$ is canonically embedded as a subring of $\left[\left[R^{S, \leq}\right]\right]$, and for each $f \in\left[\left[R^{S, \leq}\right]\right]$, and $r \in R$, $f \cdot r=f \cdot C_{r}^{0}$.

Given a ring $R$ we use $\operatorname{nil}(R)$ to denote the set of all nilpotent elements of $R$. For a subset $X$ of $R, r_{R}(X)=\{a \in R \mid X a=0\}$ and $l_{R}(X)=\{a \in R \mid$ $a X=0\}$ will stand for the right and left annihilator of $X$ in $R$ respectively. Due to Marks [14], a ring $R$ is called $N I$ if $\operatorname{nil}(R)$ forms an ideal. A ring $R$ is called reduced if it has no nonzero nilpotent elements, and a ring $R$ is called semicommutative if for all $a, b \in R, a b=0$ implies $a R b=0$. An ideal $I \subseteq R$ is said to be nilpotent if $I^{n}=0$ for some natural number $n$.

In recent years, Ribenboim [17-19] and Zhongkui Liu [11-13] have carried out an extensive study of generalized power series rings. In this note we continue the study of generalized power series rings. Firstly, as a generalization of the right (left) annihilator, we introduce a notion of a weak annihilator of a subset in a ring. Next, we investigate various weak annihilator properties of the rings of generalized power series. Consequently, several known results 
such as Ribenboim [17, 3.4] and Scott Annin [2, Theorem 5.2] and Hirano [8, Proposition 3.1] are generalized to a more general setting.

\section{On weak annihilator}

In this section, we first briefly develop the definition of the weak annihilator of a subset in a ring $R$. Also we provide several basic results. Next we discuss some weak annihilator properties of generalized power series rings.

Definition 2.1. Let $R$ be a ring. For a subset $X$ of the ring $R$, we define $N_{R}(X)=\{a \in R \mid x a \in \operatorname{nil}(R)$ for all $x \in X\}$, which is called a weak annihilator of $X$ in $R$. If $X$ is singleton, say $X=\{r\}$, we use $N_{R}(r)$ in place of $N_{R}(\{r\})$.

Obviously, for any subset $X$ of a $\operatorname{ring} R, N_{R}(X)=\{a \in R \mid x a \in \operatorname{nil}(R)$ for all $x \in X\}=\{b \in R \mid b x \in \operatorname{nil}(R)$ for all $x \in X\}, r_{R}(X) \subseteq N_{R}(X)$ and $l_{R}(X) \subseteq N_{R}(X)$. For example, let $\mathbb{Z}$ be the ring of integers and $T_{2}(\mathbb{Z})$ the $2 \times 2$ upper triangular matrix ring over $\mathbb{Z}$. We consider the subset $\left.X=\left\{\begin{array}{ll}2 & 0 \\ 0 & 2\end{array}\right)\right\}$. Then $r_{T_{2}(\mathbb{Z})}(X)=l_{T_{2}(\mathbb{Z})}(X)=0$, but $N_{T_{2}(\mathbb{Z})}(X)=\left\{\left(\begin{array}{cc}0 & m \\ 0 & 0\end{array}\right) \mid m \in \mathbb{Z}\right\}$. Thus $r_{T_{2}(\mathbb{Z})}(X) \subsetneq N_{T_{2}(\mathbb{Z})}(X)$ and $l_{T_{2}(\mathbb{Z})}(X) \subsetneq N_{T_{2}(\mathbb{Z})}(X)$. If $R$ is reduced, then $r_{R}(X)=N_{R}(X)=l_{R}(X)$ for any subset $X$ of $R$. It is easy to see that for any subset $X \subseteq R, N_{R}(X)$ is an ideal of $R$ in case $\operatorname{nil}(R)$ is an ideal.

Proposition 2.2. Let $X, Y$ be subsets of $R$. Then we have the following:

(1) $X \subseteq Y$ implies $N_{R}(X) \supseteq N_{R}(Y)$.

(2) $X \subseteq N_{R}\left(N_{R}(X)\right)$.

(3) $N_{R}(X)=N_{R}\left(N_{R}\left(N_{R}(X)\right)\right)$.

Proof. (1) and (2) are really easy.

(3) Applying (2) to $N_{R}(X)$, we obtain $N_{R}(X) \subseteq N_{R}\left(N_{R}\left(N_{R}(X)\right)\right)$. Since $X \subseteq N_{R}\left(N_{R}(X)\right)$, we have $N_{R}(X) \supseteq N_{R}\left(N_{R}\left(N_{R}(X)\right)\right)$ by (1). Therefore we get $N_{R}(X)=N_{R}\left(N_{R}\left(N_{R}(X)\right)\right)$.

Proposition 2.3. Let $R$ be a subring of $S$. Then for any subset $X$ of $R$, we have $N_{R}(X)=N_{S}(X) \cap R$.

Proof. Let $r \in N_{R}(X)$. Then $r \in R$ and $x r \in \operatorname{nil}(R)$ for each $x \in X$, and so $x r \in \operatorname{nil}(S)$ for each $x \in X$. Hence $r \in N_{S}(X) \cap R$ and so $N_{R}(X) \subseteq N_{S}(X) \cap R$. Assume that $a \in N_{S}(X) \cap R$. Then $a \in R$ and $x a \in \operatorname{nil}(S)$ for each $x \in X$. Note that $X \subseteq R$. We have $x a \in \operatorname{nil}(R)$ for each $x \in X$. Thus $a \in N_{R}(X)$ and so $N_{R}(X) \supseteq N_{S}(X) \cap R$. Therefore $N_{R}(X)=N_{S}(X) \cap R$.

Lemma 2.4. Let $R$ be an $N I$ ring and $a, b \in R$. Then $a b \in \operatorname{nil}(R)$ implies arb $\in \operatorname{nil}(R)$ for every $r \in R$.

Proof. Since $\operatorname{nil}(R)$ of an $N I$ ring is an ideal, for every $r \in R, a b \in \operatorname{nil}(R) \Rightarrow$ ba $\in \operatorname{nil}(R) \Rightarrow \operatorname{bar} \in \operatorname{nil}(R) \Rightarrow \operatorname{arb} \in \operatorname{nil}(R)$. 
Proposition 2.5. Let $R$ be an $N I$ ring and nil $(R)$ nilpotent, $S$ a cancellative torsion-free monoid, $\leq$ a strict order on $S$ and $f \in\left[\left[R^{S, \leq}\right]\right]$. Then $f \in \operatorname{nil}\left(\left[\left[R^{S, \leq}\right]\right]\right)$ if and only if $f(s) \in \operatorname{nil}(R)$ for every $s \in S$.

Proof. $(\Rightarrow)$ Observe that $R / \operatorname{nil}(R)$ is reduced and hence S-Armendariz in the sense of whenever $f, g \in\left[\left[R^{S, \leq}\right]\right]$ satisfy $f g=0$, then $f(u) g(v)=0$ for any $u, v \in S$ by [13, Lemma 3.1]. Suppose that $f^{k}=0$ for some positive integer $k$. Then if we denote by $\bar{f}$ the corresponding generalized power series of $f$ in $\left[\left[(R / \operatorname{nil}(R))^{S, \leq]]}, \bar{f}^{k}=\overline{0}\right.\right.$. Since $R / \operatorname{nil}(R)$ is S-Armendariz, $\overline{f(s)}^{k}=\overline{0}$ for any $s \in S$ by [13, Proposition 3.2]. Hence $f(s) \in \operatorname{nil}(R)$ for any $s \in S$.

$(\Leftarrow)$ Assume that $f(s) \in \operatorname{nil}(R)$ for every $s \in S$. Then $f \in\left[\left[\operatorname{nil}(R)^{S, \leq}\right]\right]$ where $\left[\left[\operatorname{nil}(R)^{S, \leq}\right]\right]=\left\{f \in\left[\left[R^{S, \leq}\right]\right] \mid f(s) \in \operatorname{nil}(R), s \in S\right\}$ is an ideal of $\left[\left[R^{S, \leq}\right]\right]$. Since $\operatorname{nil}(R)$ is nilpotent, there exists some positive integer $k$ such that $(\operatorname{nil}(R))^{k}=0$. Then it is easy to see that $\left(\left[\left[\operatorname{nil}(R)^{S, \leq]]}\right)^{k}=0\right.\right.$. Hence we obtain $f^{k}=0$. Therefore $f \in \operatorname{nil}\left(\left[\left[R^{S, \leq}\right]\right]\right)$.

Following Proposition 2.5, we obtain that if $R$ is an $N I$ ring and $\operatorname{nil}(R)$ nilpotent, $S$ a cancellative torsion-free monoid, $\leq$ a strict order on $S$, then the generalized power series $\operatorname{ring}\left[\left[R^{S, \leq}\right]\right]$ is an $N I \operatorname{ring}$ and $\operatorname{nil}\left(\left[\left[R^{S, \leq}\right]\right]\right)=$ $\left[\left[\operatorname{nil}(R)^{S, \leq}\right]\right]$.

It was proved in Ribenboim $[17,3.3]$ that if $R$ is a Noetherian commutative ring, $(S, \leq)$ a cancellative torsion-free strictly ordered monoid and $f \in\left[\left[R^{S, \leq}\right]\right]$, then $f \in \operatorname{nil}\left(\left[\left[R^{S,} \leq\right]\right]\right)$ if and only if $f(s) \in \operatorname{nil}(R)$ for all $s \in S$. In the following, we show that the same is true even if $R$ is noncommutative.

Corollary 2.6. Let $R$ be a right Noetherian semicommutative ring, $(S, \leq)$ a cancellative torsion-free strictly ordered monoid and $f \in\left[\left[R^{S, \leq}\right]\right]$. Then $f \in$ $\operatorname{nil}\left(\left[\left[R^{S,} \leq\right]\right]\right)$ if and only if $f(s) \in \operatorname{nil}(R)$ for all $s \in S$.

Proof. It suffices to show that $\operatorname{nil}(R)$ is nilpotent. Since $R$ is a right Noetherian ring, we can find $a_{1}, a_{2}, \ldots, a_{n} \in \operatorname{nil}(R)$ such that $\operatorname{nil}(R)$ is generated by $a_{1}$, $a_{2}, \ldots, a_{n}$. Let $k \geq 1$ be such that $a_{i}^{k}=0$ for all $1 \leq i \leq n$. We claim that $(\operatorname{nil}(R))^{n k+1}=0$. Consider a product

$\left(a_{1} r_{11}+a_{2} r_{12}+\cdots+a_{n} r_{1 n}\right) \cdots\left(a_{1} r_{(n k+1) 1}+a_{2} r_{(n k+1) 2}+\cdots+a_{n} r_{(n k+1) n}\right)$

of $n k+1$ elements in $\operatorname{nil}(R)$. When this product is expanded, each term in it is a product of $2(n k+1)$ elements, $n k+1$ elements from the set $\left\{a_{1}, a_{2}, \ldots, a_{n}\right\}$, and $n k+1$ elements from the set $\left\{r_{i j} \mid 1 \leq i \leq n k+1,1 \leq j \leq n\right\}$. Consider each term

$$
a_{v_{1}} r_{v_{1}} a_{v_{2}} r_{v_{2}} \cdots a_{v_{n k+1}} r_{v_{n k+1}},
$$

where $a_{v_{1}}, a_{v_{2}}, \ldots, a_{v_{n k+1}} \in\left\{a_{1}, a_{2}, \ldots, a_{n}\right\}$ and $r_{v_{j}} \in R$ for all $1 \leq j \leq n k+1$. We will show that

$$
a_{v_{1}} r_{v_{1}} a_{v_{2}} r_{v_{2}} \cdots a_{v_{n k+1}} r_{v_{n k+1}}=0
$$

If the number of $a_{1}$ in $a_{v_{1}} r_{v_{1}} a_{v_{2}} r_{v_{2}} \cdots a_{v_{n k+1}} r_{v_{n k+1}}$ is greater than $k$, then we can write

$$
a_{v_{1}} r_{v_{1}} a_{v_{2}} r_{v_{2}} \cdots a_{v_{n k+1}} r_{v_{n k+1}}
$$


as

$$
b_{1} a_{1}^{j_{1}} b_{2} a_{1}^{j_{2}} \cdots b_{p} a_{1}^{j_{p}} b_{p+1}
$$

where $j_{1}+j_{2}+\cdots+j_{p}>k$ and $b_{q} \in R$ for all $1 \leq q \leq p+1$. Since $R$ is a semicommutative ring and $a_{1}^{j_{1}+j_{2}+\cdots+j_{p}}=0$, it is easy to see that $b_{1} a_{1}^{j_{1}} b_{2} a_{1}^{j_{2}} \cdots b_{p} a_{1}^{j_{p}} b_{p+1}=0$, and so $a_{v_{1}} r_{v_{1}} a_{v_{2}} r_{v_{2}} \cdots a_{v_{n k+1}} r_{v_{n k+1}}=0$. If the number of $a_{i}$ in $a_{v_{1}} r_{v_{1}} a_{v_{2}} r_{v_{2}} \cdots a_{v_{n k+1}} r_{v_{n k+1}}$ is greater than $k$, then similar discuss yields that $a_{v_{1}} r_{v_{1}} a_{v_{2}} r_{v_{2}} \cdots a_{v_{n k+1}} r_{v_{n k+1}}=0$. Thus each term is zero, and so

$\left(a_{1} r_{11}+a_{2} r_{12}+\cdots+a_{n} r_{1 n}\right) \cdots\left(a_{1} r_{(n k+1) 1}+a_{2} r_{(n k+1) 2}+\cdots+a_{n} r_{(n k+1) n}\right)=0$.

Therefore $\operatorname{nil}(R)$ is nilpotent, as required.

Proposition 2.7. Let $R$ be an NI ring and nil $(R)$ nilpotent, $(S, \leq)$ a cancellative torsion-free strictly ordered monoid, $f, g, h \in\left[\left[R^{S,} \leq\right]\right]$ and $r \in R$. Then we have the following:

(1) $f g \in \operatorname{nil}\left(\left[\left[R^{S, \leq}\right]\right]\right) \Longleftrightarrow f(u) g(v) \in \operatorname{nil}(R)$ for all $u, v \in S$.

(2) $f g r=f g C_{r}^{0} \in \operatorname{nil}\left(\left[\left[R^{S,} \leq\right]\right]\right) \Longleftrightarrow f(u) g(v) r \in \operatorname{nil}(R)$ for all $u, v \in S$.

(3) $f g h \in \operatorname{nil}\left(\left[\left[R^{S, \leq}\right]\right]\right) \Longleftrightarrow f(u) g(v) h(w) \in \operatorname{nil}(R)$ for all $u, v, w \in S$.

Proof. (1) Suppose that $f g \in \operatorname{nil}\left(\left[\left[R^{S,} \leq\right]\right]\right)$. Then $f g \in\left[\left[\operatorname{nil}(R)^{S, \leq]]}\right.\right.$ by Proposition 2.5. Thus $\bar{f} \bar{g}=\overline{0}$ where $\bar{f}, \bar{g}$ are the corresponding generalized power series of $f, g$ in $\left[\left[(R / \operatorname{nil}(R))^{S, \leq]}\right]\right.$. Since $R / \operatorname{nil}(R)$ is S-Armendariz, $\overline{f(u)} \overline{g(v)}=\overline{0}$ for any $u, v \in S$. Hence $f(u) g(v) \in \operatorname{nil}(R)$ for any $u, v \in S$. Conversely, let $f, g \in\left[\left[R^{S, \leq}\right]\right]$ be such that $f(u) g(v) \in \operatorname{nil}(R)$ for any $u, v \in S$. Then $(f g)(s) \in \operatorname{nil}(R)$ since $\operatorname{nil}(R)$ is an ideal of $R$. Hence $f g \in \operatorname{nil}\left(\left[\left[R^{S, \leq}\right]\right]\right)$ by Proposition 2.5.

(2) $(\Rightarrow)$ Suppose that $f g C_{r}^{0}=f\left(g C_{r}^{0}\right) \in \operatorname{nil}\left(\left[\left[R^{S, \leq}\right]\right]\right)$. Then for any $u$, $v \in S$, by (1), we obtain $f(u)\left(g C_{r}^{0}\right)(v)=f(u) g(v) r \in \operatorname{nil}(R)$.

$(\Leftarrow)$ Suppose that $f(u) g(v) r \in \operatorname{nil}(R)$ for all $u, v \in S$. We show that $f g r=f g C_{r}^{0} \in \operatorname{nil}\left(\left[\left[R^{S, \leq}\right]\right]\right)$. For any $s \in S$, we have

$$
\left(f g C_{r}^{0}\right)(s)=\sum_{(u, v) \in X_{s}(f, g)} f(u) g(v) r,
$$

and $f(u) g(v) r \in \operatorname{nil}(R)$ for all $u, v \in S$ implies that $\left(f g C_{r}^{0}\right)(s) \in \operatorname{nil}(R)$. Thus by Proposition $2.5, f g C_{r}^{0} \in \operatorname{nil}\left(\left[\left[R^{S, \leq}\right]\right]\right)$.

(3) It suffices to show $(\Rightarrow)$. Suppose that $f g h \in \operatorname{nil}\left(\left[\left[R^{S, \leq]]}\right)\right.\right.$. Then from $f g h=(f g) h \in \operatorname{nil}\left(\left[\left[R^{S, \leq}\right]\right]\right)$, it follows that $(f g)(p) h(w) \in \operatorname{nil}(R)$ for each $p$, $w \in S$. Now consider $(f g) C_{h(w)}^{0}$. Since $\operatorname{supp}\left(C_{h(w)}^{0}\right)=\{0\}$ and $C_{h(w)}^{0}(0)=$ $h(w)$, thus, by $(1)$, we obtain $(f g) C_{h(w)}^{0} \in \operatorname{nil}\left(\left[\left[R^{S, \leq}\right]\right]\right)$ for each $w \in S$. Now by (2), we obtain $f(u) g(v) h(w) \in \operatorname{nil}(R)$ for all $u, v, w \in S$.

Corollary 2.8. Let $R$ be a right Noetherian semicommutative ring, $(S, \leq)$ a cancellative torsion-free strictly ordered monoid, $f, g, h \in\left[\left[R^{S, \leq}\right]\right]$ and $r \in R$. Then we have the following:

(1) $f g \in \operatorname{nil}\left(\left[\left[R^{S, \leq}\right]\right]\right) \Longleftrightarrow f(u) g(v) \in \operatorname{nil}(R)$ for all $u, v \in S$. 
(2) $f g r=f g C_{r}^{0} \in \operatorname{nil}\left(\left[\left[R^{S, \leq}\right]\right]\right) \Longleftrightarrow f(u) g(v) r \in \operatorname{nil}(R)$ for all $u, v \in S$.

(3) $f g h \in \operatorname{nil}\left(\left[\left[R^{S, \leq}\right]\right]\right) \Longleftrightarrow f(u) g(v) h(w) \in \operatorname{nil}(R)$ for all $u, v, w \in S$.

Proof. By analogy with the proof of Proposition 2.7, we can complete the proof.

Hirano observed relations between annihilators in a ring $R$ and annihilators in $R[x]$ (see [8]). In this note, we investigate the relations between weak annihilators in a ring $R$ and weak annihilators in $\left[\left[R^{S, \leq}\right]\right]$. Given a ring $R$, we define

$$
N A n n_{R}\left(2^{R}\right)=\left\{N_{R}(U) \mid U \subseteq R\right\},
$$

and

$$
N A n n_{\left[\left[R^{S, \leq]]}\right.\right.}\left(2^{\left[\left[R^{S,} \leq\right]\right]}\right)=\left\{N_{\left[\left[R^{S, \leq]}\right]\right.}(V) \mid V \subseteq\left[\left[R^{S, \leq]}\right]\right\} .\right.
$$

For a generalized power series $f \in\left[\left[R^{S, \leq}\right]\right]$, let $C_{f}$ denote the set $\{f(s) \mid s \in S\}$ and for a subset $V$ of $\left[\left[R^{S, \leq}\right]\right]$, let $C_{V}$ denote the set $\cup_{f \in V} C_{f}$.

Given a subset $U \subseteq R$, let $\left[\left[U^{S, \leq}\right]\right]$ denote the set $\left\{f \in\left[\left[R^{S, \leq}\right]\right] \mid f(s) \in\right.$ $U, s \in S\}$. Then we can construct a map

$$
\phi: \quad N A n n_{R}\left(2^{R}\right) \longrightarrow N A n n_{\left[\left[R^{S, \leq]]}\right.\right.}\left(2^{\left[\left[R^{S, \leq}\right]\right]}\right)
$$

defined by $\phi\left(N_{R}(U)\right)=N_{\left[\left[R^{S, \leq]]}\right.\right.}\left(\left[\left[U^{S, \leq}\right]\right]\right)$ for any $N_{R}(U) \in N A n n_{R}\left(2^{R}\right)$.

Proposition 2.9. Let $R$ be an $N I$ ring and nil $(R)$ nilpotent, $(S, \leq)$ a cancellative torsion-free strictly ordered monoid. Then

$$
\phi: N A n n_{R}\left(2^{R}\right) \longrightarrow N A n n_{\left[\left[R^{S, \leq]]}\right.\right.}\left(2^{\left[\left[R^{S, \leq}\right]\right]}\right)
$$

defined by $\phi\left(N_{R}(U)\right)=N_{\left[\left[R^{S, \leq]]}\right.\right.}\left(\left[\left[U^{S, \leq}\right]\right]\right)$ for any $N_{R}(U) \in N A n n_{R}\left(2^{R}\right)$ is bijective.

Proof. We show that $\phi$ is injective. Suppose $N_{R}(U) \in N A n n_{R}\left(2^{R}\right), N_{R}\left(U^{\prime}\right) \in$ $N A n n_{R}\left(2^{R}\right)$ and $N_{R}(U) \neq N_{R}\left(U^{\prime}\right)$. Without loss of generality, we may assume that there exists $r \in R$ such that $r \in N_{R}(U)$, and $r \notin N_{R}\left(U^{\prime}\right)$. Then it is easy to see that $C_{r}^{0} \in N_{\left[\left[R^{S, \leq}\right]\right]}\left(\left[\left[U^{S, \leq}\right]\right]\right)$ and $C_{r}^{0} \notin N_{\left[\left[R^{S, \leq}\right]\right]}\left(\left[\left[\left(U^{\prime}\right)^{S, \leq}\right]\right]\right)$, and

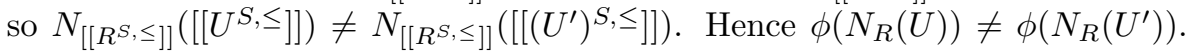
Therefore $\phi$ is injective.

Now we show that $\phi$ is surjective. For any

$$
N_{\left[\left[R^{S, \leq]]}\right.\right.}(V) \in N A n n_{\left[\left[R^{S, \leq]]}\right.\right.}\left(2^{\left[\left[R^{S, \leq]]}\right.\right.}\right), V \subseteq\left[\left[R^{S, \leq]]},\right.\right.
$$

then $N_{R}\left(C_{V}\right) \in N A n n_{R}\left(2^{R}\right)$. To show $\phi$ is surjective, it suffices to show

$$
\phi\left(N_{R}\left(C_{V}\right)\right)=N_{\left[\left[R^{S, \leq}\right]\right]}\left(\left[\left[\left(C_{V}\right)^{S, \leq}\right]\right]\right)=N_{\left[\left[R^{S, \leq]]}\right.\right.}(V) .
$$

Since $V \subseteq\left[\left[\left(C_{V}\right)^{S, \leq]}\right], N_{\left[\left[R^{S,} \leq\right]\right]}\left(\left[\left[\left(C_{V}\right)^{S, \leq}\right]\right]\right) \subseteq N_{\left[\left[R^{S,} \leq\right]\right]}(V)\right.$ is clear. Now we show that $N_{\left[\left[R^{S, \leq]]}\right.\right.}(V) \subseteq N_{\left[\left[R^{S, \leq]]}\right.\right.}\left(\left[\left[\left(C_{V}\right)^{S, \leq]])}\right.\right.\right.$. Assume that $f \in N_{\left[\left[R^{S, \leq]]}\right.\right.}(V)$. Then $g f \in \operatorname{nil}\left(\left[\left[R^{S, \leq}\right]\right]\right)$ for all $g \in V$. By Proposition 2.7, we obtain $g(u) f(v) \in$ $\operatorname{nil}(R)$ for all $u, v \in S$, and so $f(v) \in N_{R}\left(C_{V}\right)$ for every $v \in S$. Then for each $h \in\left[\left[\left(C_{V}\right)^{S, \leq}\right]\right]$, by Proposition 2.7 , it is easy to see that $h f \in \operatorname{nil}\left(\left[\left[R^{S, \leq}\right]\right]\right)$, 
and so $f \in N_{\left[\left[R^{S, \leq]]}\right.\right.}\left(\left[\left[\left(C_{V}\right)^{S, \leq}\right]\right]\right)$. Hence $N_{\left[\left[R^{S, \leq \leq]]}\right.\right.}(V) \subseteq N_{\left[\left[R^{S, \leq]]}\right.\right.}\left(\left[\left[\left(C_{V}\right)^{S, \leq}\right]\right]\right)$. Therefore $N_{\left[\left[R^{S, \leq]]}\right.\right.}(V)=N_{\left[\left[R^{S, \leq]]}\right.\right.}\left(\left[\left[\left(C_{V}\right)^{S, \leq]]}\right)=\phi\left(N_{R}\left(C_{V}\right)\right)\right.\right.$, as required.

A ring $R$ is called right zip provided that the right annihilator $r_{R}(X)$ of a subset $X$ of $R$ is zero, then there exists a finite subset $Y$ of $X$, such that $r_{R}(Y)=0$. Beachy and Blair [4] showed that if $R$ is a commutative zip ring, then the polynomial ring $R[x]$ over $R$ is a zip ring. Hong et al. [9, Theorem 11] proved that $R$ is a right (left) zip ring if and only if $R[x]$ is a right (left) zip ring when $R$ is an Armendariz ring. As a generalization of zip rings, in [15], L. Ouyang introduced the notion of weak zip rings and showed that if $R$ is an $(\alpha, \delta)$-compatible and reversible ring, then $R$ is weak zip if and only if the Ore extension $R[x ; \alpha, \delta]$ is weak zip. In the following, we investigate the weak zip property of rings of generalized power series.

Definition 2.10. A ring $R$ is called a weak zip ring provided that for any subset $X$ of $R$, if $N_{R}(X) \subseteq \operatorname{nil}(R)$, then there exists a finite subset $Y \subseteq X$ such that $N_{R}(Y) \subseteq \operatorname{nil}(R)$.

Obviously, all reduced zip rings are weak zip, and if $R$ is a weak zip ring, then so is the $n \times n$ upper triangular matrix ring over $R$. Further examples and some properties of weak zip rings are given in [15].

Proposition 2.11. Let $R$ be an $N I$ ring and nil $(R)$ nilpotent, $(S, \leq)$ a cancellative torsion-free strictly ordered monoid. Then $R$ is weak zip if and only if $\left[\left[R^{S, \leq}\right]\right]$ is weak zip.

Proof. Assume that $R$ is weak zip and $V$ a subset of $\left[\left[R^{S, \leq}\right]\right]$ with $N_{\left[\left[R^{S, \leq}\right]\right]}(V) \subseteq$ $\operatorname{nil}\left(\left[\left[R^{S, \leq}\right]\right]\right)$. Now we show that $N_{R}\left(C_{V}\right) \subseteq \operatorname{nil}(R)$. If $r \in N_{R}\left(C_{V}\right)$, then $a r \in \operatorname{nil}(R)$ for all $a \in C_{V}$. So for any $f \in V$ and any $s \in S$,

$$
(f r)(s)=\left(f C_{r}^{0}\right)(s)=f(s) r \in \operatorname{nil}(R),
$$

and so by Proposition $2.5, f r \in \operatorname{nil}\left(\left[\left[R^{S, \leq}\right]\right]\right)$. Hence

$$
r \in N_{\left[\left[R^{S,} \leq\right]\right]}(V) \subseteq \operatorname{nil}\left(\left[\left[R^{S, \leq}\right]\right]\right) .
$$

Thus $r \in \operatorname{nil}(R)$ and this implies $N_{R}\left(C_{V}\right) \subseteq \operatorname{nil}(R)$. Since $R$ is weak zip, there exists a finite subset $Y_{0}=\left\{q_{1}, \ldots, q_{m}\right\} \subseteq C_{V}$, such that $N_{R}\left(Y_{0}\right) \subseteq$ $\operatorname{nil}(R)$. Let $f_{i}$ be an element of $V$ such that $f_{i}\left(s_{i}\right)=q_{i}$ for some $s_{i} \in S$, $i=1,2, \ldots, m$. Let $V_{0}=\left\{f_{1}, f_{2}, \ldots, f_{m}\right\}$. Then $V_{0}$ is a finite subset of $V$, and $C_{V_{0}} \supseteq Y_{0}$. So $N_{R}\left(C_{V_{0}}\right) \subseteq N_{R}\left(Y_{0}\right) \subseteq \operatorname{nil}(R)$. Now we show that $N_{\left[\left[R^{S, \leq]]}\right.\right.}\left(V_{0}\right) \subseteq$ $\operatorname{nil}\left(\left[\left[R^{S, \leq}\right]\right]\right)$. Suppose $g \in N_{\left[\left[R^{S, \leq]}\right.\right.}\left(V_{0}\right)$. Then $f g \in \operatorname{nil}\left(\left[\left[R^{S, \leq}\right]\right]\right)$ for all $f \in V_{0}$. By Proposition 2.7, we obtain $f(u) g(v) \in \operatorname{nil}(R)$ for all $u, v \in S$. Hence $g(v) \in$ $N_{R}\left(C_{V_{0}}\right) \subseteq \operatorname{nil}(R)$ for all $v \in S$, and so by Proposition $2.5, g \in \operatorname{nil}\left(\left[\left[R^{S, \leq}\right]\right]\right)$. Hence $N_{\left[\left[R^{S, \leq]}\right.\right.}\left(V_{0}\right) \subseteq \operatorname{nil}\left(\left[\left[R^{S, \leq}\right]\right]\right)$. Therefore $\left[\left[R^{S, \leq}\right]\right]$ is weak zip.

Conversely, let $Y \subseteq R$ with $N_{R}(Y) \subseteq \operatorname{nil}(R)$. If $f \in N_{\left[\left[R^{S, \leq]]}\right.\right.}(Y)$, then $y f=C_{y}^{0} f \in \operatorname{nil}\left(\left[\left[R^{S, \leq}\right]\right]\right)$ for all $y \in Y$, and so $y f(s) \in \operatorname{nil}(R)$ for all $y \in Y$, and $s \in S$. Thus $f(s) \in N_{R}(Y) \subseteq \operatorname{nil}(R)$ for all $s \in S$. By Proposition 2.5, 
$f \in \operatorname{nil}\left(\left[\left[R^{S, \leq]])}\right)\right.\right.$. Hence $N_{\left[\left[R^{S,} \leq\right]\right]}(Y) \subseteq \operatorname{nil}\left(\left[\left[R^{S, \leq]}\right]\right)\right.$. Since $\left[\left[R^{S, \leq]]}\right.\right.$ is weak zip, there exists a finite subset $Y_{0} \subseteq Y$ such that $N_{\left[\left[R^{S,} \leq\right]\right]}\left(Y_{0}\right) \subseteq \operatorname{nil}\left(\left[\left[R^{S, \leq}\right]\right]\right)$. Hence $N_{R}\left(Y_{0}\right)=N_{\left[\left[R^{S,} \leq\right]\right]}\left(Y_{0}\right) \cap R \subseteq \operatorname{nil}\left(\left[\left[R^{S, \leq}\right]\right]\right) \cap R=\operatorname{nil}(R)$. Therefore $R$ is weak zip.

Following Lambek [10], a ring $R$ is called symmetric if $a b c=0$ implies $a c b=0$ for all $a, b, c \in R$. It is obvious that commutative rings are symmetric. Reduced rings are symmetric by the results of Anderson and Camillo [1], but there are many nonreduced commutative (so symmetric) rings. As a generalization of symmetric rings, L. Ouyang introduced the notion of weak symmetric rings and showed that if $R$ is an $(\alpha, \delta)$-compatible and reversible ring, then $R$ is weak symmetric if and only if the Ore extension $R[x ; \alpha, \delta]$ is weak symmetric [16]. In the following, we investigate the weak symmetric property of the rings of generalized power series.

Definition 2.12. A ring $R$ is called a weak symmetric ring if $a b c \in \operatorname{nil}(R) \Rightarrow$ $a c b \in \operatorname{nil}(R)$ for all $a, b, c \in R$.

Proposition 2.13. Let $R$ be an $N I$ ring and nil $(R)$ nilpotent, $(S, \leq)$ a cancellative torsion-free strictly ordered monoid. Then $R$ is weak symmetric if and only if $\left[\left[R^{S, \leq}\right]\right]$ is weak symmetric.

Proof. Since any subring of a weak symmetric ring is again a weak symmetric ring, it suffices to show that if $R$ is a weak symmetric ring, then so is $\left[\left[R^{S, \leq}\right]\right]$. Let $f, g, h \in\left[\left[R^{S, \leq}\right]\right]$ be such that $f g h \in \operatorname{nil}\left(\left[\left[R^{S,} \leq\right]\right]\right)$. By Proposition 2.7, we have $f(u) g(v) h(w) \in \operatorname{nil}(R)$ for all $u, v, w \in S$, and so $f(u) h(w) g(v) \in \operatorname{nil}(R)$ for all $u, w, v \in S$ since $R$ is weak symmetric. Hence $f h g \in \operatorname{nil}\left(\left[\left[R^{S, \leq}\right]\right]\right)$ by Proposition 2.7. Therefore $\left[\left[R^{S, \leq}\right]\right]$ is a weak symmetric ring.

The following corollary will give more examples of weak zip rings and weak symmetric rings.

Corollary 2.14. Let $\left(S_{1}, \leq_{1}\right),\left(S_{2}, \leq_{2}\right), \ldots,\left(S_{n}, \leq_{n}\right)$ be cancellative torsionfree strictly ordered monoids. Denote by (lex $\leq$ ) and (revlex $\leq$ ) the lexicographic order, the reverse lexicographic order, respectively, on the monoid $S_{1} \times S_{2} \times \cdots \times S_{n}$. If $R$ is an $N I$ ring and nil $(R)$ nilpotent, then we have the following:

(1) $R$ is weak zip $\Leftrightarrow\left[\left[R^{S_{1} \times S_{2} \times \cdots \times S_{n},(l e x \leq)}\right]\right]$ is weak zip.

(2) $R$ is weak zip $\Leftrightarrow\left[\left[R^{S_{1} \times S_{2} \times \cdots \times S_{n}},(\right.\right.$ revlex $\left.\left.\leq)\right]\right]$ is weak zip.

(3) $R$ is weak symmetric $\Leftrightarrow\left[\left[R^{S_{1} \times S_{2} \times \cdots \times S_{n},(l e x \leq)}\right]\right]$ is weak symmetric.

(4) $R$ is weak symmetric $\Leftrightarrow\left[\left[R^{S_{1} \times S_{2} \times \cdots \times S_{n},(\text { revlex } \leq)}\right]\right]$ is weak symmetric.

Proof. It is easy to see that $\left(S_{1} \times S_{2} \times \cdots \times S_{n},(\right.$ lex $\left.\leq)\right)$ and $\left(S_{1} \times S_{2} \times \cdots \times\right.$ $S_{n},($ revlex $\left.\leq)\right)$ are cancellative torsion-free strictly ordered monoids. Therefore we complete the proofs of (1), (2) by Proposition 2.11, and (3), (4) by Proposition 2.13. 


\section{Weak associated primes}

Given a right $R$-module $N_{R}$, the right annihilator of $N_{R}$ is denoted by $r_{R}\left(N_{R}\right)=\{a \in R \mid N a=0\}$. We say that $N_{R}$ is prime if $N_{R} \neq 0$, and $r_{R}\left(N_{R}\right)=r_{R}\left(N_{R}^{\prime}\right)$ for every nonzero submodule $N_{R}^{\prime} \subseteq N_{R}$ (see [2], [3]). Let $M_{R}$ be a right $R$-module, an ideal $\wp$ of $R$ is called an associated prime of $M_{R}$ if there exists a prime submodule $N_{R} \subseteq M_{R}$ such that $\wp=r_{R}\left(N_{R}\right)$. The set of associated primes of $M_{R}$ is denoted by $A s s\left(M_{R}\right)$ (see [2], [3]). Associated primes are well-known in commutative algebra for their important role in the primary decomposition, and has attracted a lot of attention in recent years. In [5], Brewer and Heinzer used localization theory to prove that, over a commutative ring $R$, the associated primes of the polynomial ring $R[x]$ (viewed as a module over itself) are all extended: that is, every $\wp \in A s s(R[x])$ may be expressed as $\wp=\wp_{0}[x]$, where $\wp_{0}=\wp \cap R \in A s s(R)$. Using results of $\mathrm{R}$. C. Shock in [20] on good polynomials, C. Faith has provided a new proof in [7] of the same result which does not rely on localization or other tools from commutative algebra. In [3], Scott Annin showed that Brewer and Heinzer's result still holds in the more general setting of a polynomial module $M[x]$ over an Ore extension ring $R[x ; \alpha, \delta]$, with possibly noncommutative base $R$. So the properties of associated primes over a commutative ring can be profitably generalized to a noncommutative setting as well.

Motivated by the results in [2], [3], [7], [20], in this section, we first introduce the notion of weak associated primes, which is a generalization of associated primes. We next describe all weak associated primes of the generalized power series ring $\left[\left[R^{S,} \leq\right]\right]$ in terms of the weak associated primes of the ring $R$.

Definition 3.1. Let $I$ be a right ideal of a nonzero ring $R$. We say that $I$ is an $R$-prime ideal if $I \nsubseteq \operatorname{nil}(R)$ and $N_{R}(I)=N_{R}\left(I^{\prime}\right)$ for every right ideal $I^{\prime} \subseteq I$ and $I^{\prime} \nsubseteq \operatorname{nil}(R)$.

Definition 3.2. Let $\operatorname{nil}(R)$ be an ideal of a ring $R$. An ideal $\wp$ of $R$ is called a weak associated prime of $R$ if there exists an $R$-prime ideal $I$ such that $\wp=N_{R}(I)$. The set of weak associated primes of $R$ is denoted by $N A s s(R)$.

Example 3.3. Let $R$ be a domain and let

$$
R_{n}=\left\{\left(\begin{array}{llll}
a & a_{12} & \cdots & a_{1 n} \\
0 & a & \cdots & a_{2 n} \\
\cdots & \cdots & \cdots & \cdots \\
0 & 0 & \cdots & a
\end{array}\right) \mid a, a_{i j} \in R\right\}
$$

be the subring of $n \times n$ upper triangular matrix ring. Then $\operatorname{nil}\left(R_{n}\right)$ is an ideal of $R_{n}$ and

$$
\operatorname{nil}\left(R_{n}\right)=\left\{\left(\begin{array}{llll}
0 & x_{12} & \cdots & x_{1 n} \\
0 & 0 & \cdots & x_{2 n} \\
\cdots & \cdots & \cdots & \cdots \\
0 & 0 & \cdots & 0
\end{array}\right) \mid x_{i j} \in R\right\}
$$


By a routine computations, we know that each right ideal $I \nsubseteq \operatorname{nil}\left(R_{n}\right)$ is an $R_{n}$-prime ideal, and $N A s s\left(R_{n}\right)=\left\{\operatorname{nil}\left(R_{n}\right)\right\}$.

Example 3.4. Let $k$ be any field, and consider the ring $R=\left(\begin{array}{ll}k & 0 \\ k & k\end{array}\right)$ of $2 \times 2$ lower triangular matrices over $k$. One easily checks that $\left(\begin{array}{ll}k & 0 \\ k & k\end{array}\right) \supsetneq\left(\begin{array}{ll}k & 0 \\ k & 0\end{array}\right) \supsetneq\left(\begin{array}{ll}k & 0 \\ 0 & 0\end{array}\right) \supsetneq$ $\left(\begin{array}{ll}0 & 0 \\ 0 & 0\end{array}\right)$ is a composition series for $R_{R}$. In particular, $R_{R}$ has finite length.

Next we shall determine the set $A s s(R)$. By an easy ad hoc calculation, we can write down all of the proper nonzero ideals of $R$ :

$$
\left\{m_{1}=\left(\begin{array}{ll}
0 & 0 \\
k & k
\end{array}\right), m_{2}=\left(\begin{array}{cc}
k & 0 \\
k & 0
\end{array}\right), \alpha=\left(\begin{array}{ll}
0 & 0 \\
k & 0
\end{array}\right)\right\} .
$$

Now since $\alpha^{2}=0,0$ is not a prime ideal. Moreover, since $m_{1} R m_{2} \subseteq \alpha, \alpha$ is not a prime ideal. So the only candidates for the associated primes of $R$ are the maximal ideals $m_{1}$ and $m_{2}$.

We claim that $m_{2} \notin A s s(R)$. Otherwise, there would exist a right ideal $I \supsetneq 0$ of $R$ with $m_{2}=r_{R}(I)$. So $I \cdot m_{2}=0$. Now, given $\left(\begin{array}{ll}a & 0 \\ b & c\end{array}\right) \in I$, we have $0=\left(\begin{array}{ll}a & 0 \\ b & c\end{array}\right) \cdot\left(\begin{array}{ll}1 & 0 \\ 0 & 0\end{array}\right)=\left(\begin{array}{ll}a & 0 \\ b & 0\end{array}\right)$, so $a=b=0$. Also, $0=\left(\begin{array}{ll}0 & 0 \\ 0 & c\end{array}\right) \cdot\left(\begin{array}{ll}0 & 0 \\ 1 & 0\end{array}\right)=\left(\begin{array}{ll}0 & 0 \\ c & 0\end{array}\right)$ implies that $c=0$. Thus $I=0$, a contradiction. Hence $m_{2} \notin A s s(R)$.

By virtue of $R_{R}$ being Noetherian, we know that $A s s(R) \neq 0$. Hence $\operatorname{Ass}(R)=\left\{m_{1}\right\}$.

Finally, we should determine the set of $N A s s(R)$. Clearly, $\operatorname{nil}(R)=\alpha$. Thus $\operatorname{nil}(R)$ is an ideal. Now we show that $m_{1}=N_{R}\left(m_{2}\right)$ and $m_{2}$ is a right $R$-prime ideal. Clearly, $m_{1} \subseteq N_{R}\left(m_{2}\right)$ since $m_{2} m_{1}=0$. Given $\left(\begin{array}{cc}a & 0 \\ b & c\end{array}\right) \in N_{R}\left(m_{2}\right)$, we have $\left(\begin{array}{ll}1 & 0 \\ 0 & 0\end{array}\right)\left(\begin{array}{ll}a & 0 \\ b & c\end{array}\right)=\left(\begin{array}{ll}a & 0 \\ 0 & 0\end{array}\right) \in \operatorname{nil}(R)$. Then $a=0$ and so $\left(\begin{array}{ll}a & 0 \\ b & c\end{array}\right) \in m_{1}$. Hence $m_{1}=$ $N_{R}\left(m_{2}\right)$. Next we see that $m_{2}$ is a right $R$-prime ideal. Let $n \nsubseteq \operatorname{nil}(R)$ and $n \subseteq m_{2}$. Since $N_{R}(n) \supseteq N_{R}\left(m_{2}\right)$ is clear, we now assume that $\left(\begin{array}{ll}a & 0 \\ b & c\end{array}\right) \in N_{R}(n)$,

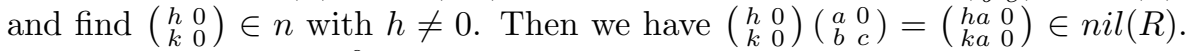
Thus $a=0$ and so $\left(\begin{array}{ll}a & 0 \\ b & c\end{array}\right) \in N_{R}\left(m_{2}\right)$. Hence we obtain $N_{R}(n)=N_{R}\left(m_{2}\right)$ and so $m_{2}$ is a right $R$-prime ideal. Thus we obtain $m_{1} \in N A s s(R)$. Similarly, we have $m_{2} \in N A s s(R)$. Therefore $N A s s(R)=\left\{m_{1}, m_{2}\right\} \neq A s s(R)$.

If $R$ is reduced, then $\wp$ is a weak associated prime of $R$ if and only if $\wp$ is an associated prime of $R$. So $N A s s(R)=A s s(R)$ in case $R$ is reduced.

In the following, unless stated otherwise, we shall always assume that $R$ is a semicommutative right Noetherian ring, and $(S, \leq)$ a strictly totally ordered monoid.

Let $f \in\left[\left[R^{S, \leq}\right]\right]$. We denote by $N(f)$ the right ideal of $R$ generated by $C_{f}=\{f(s) \mid s \in S\}$. Since $R$ is a right Noetherian ring, we can find $s_{i} \in S$, $i=1,2, \ldots, n$, and $s_{1} \leq s_{2} \leq \cdots \leq s_{n}$, such that $N(f)$ is generated by $f\left(s_{1}\right)$, $f\left(s_{2}\right), \ldots, f\left(s_{n}\right)$. Consider the $n$ elements $f\left(s_{1}\right), f\left(s_{2}\right), \ldots, f\left(s_{k-1}\right), f\left(s_{k}\right)$, $f\left(s_{k+1}\right), \ldots, f\left(s_{n}\right)$. If $f\left(s_{k}\right) \notin \operatorname{nil}(R)$, and $f\left(s_{i}\right) \in \operatorname{nil}(R)$ for all $k<i \leq n$, then we say that the weak degree of $f$ is $k$. To simplify notations, we write $N \operatorname{deg}(f)$ for the weak degree of $f$. If $f\left(s_{i}\right) \in \operatorname{nil}(R)$ for all $1 \leq i \leq n$, then we define $N \operatorname{deg}(f)=-1$. 
Definition 3.5. Let $f \in\left[\left[R^{S, \leq}\right]\right], N(f)$ is generated by $f\left(s_{1}\right), f\left(s_{2}\right), \ldots, f\left(s_{n}\right)$, $s_{i} \leq s_{j}$ if $i \leq j$, and $N \operatorname{deg}(f)=k$. If $N_{R}\left(f\left(s_{k}\right)\right) \subseteq N_{R}\left(f\left(s_{i}\right)\right)$ for all $i \leq k$, then we say that $f$ is a weak good generalized power series.

Lemma 3.6. Let $R$ be a semicommutative right Noetherian ring, $(S, \leq)$ a strictly totally ordered monoid. For any $f \notin$ nil $\left(\left[\left[R^{S, \leq}\right]\right]\right)$, there exists $r \in R$ such that $f r=f C_{r}^{0}$ is a weak good generalized power series.

Proof. Assume that the result is false, and let $f \notin \operatorname{nil}\left(\left[\left[R^{S, \leq}\right]\right]\right)$ be a counterexample of minimal weak degree $N \operatorname{deg}(f)=k \geq 1$. In particular, $f$ is not a weak good generalized power series. Suppose that $N(f)$ is generated by $f\left(s_{1}\right), f\left(s_{2}\right), \ldots, f\left(s_{n}\right)$, where $s_{i} \leq s_{j}$ if $i \leq j$. Hence there exists $i<k$ such that $N_{R}\left(f\left(s_{k}\right)\right) \nsubseteq N_{R}\left(f\left(s_{i}\right)\right)$. So we can find $b \in R$ with $f\left(s_{i}\right) b \notin n i l(R)$, and $f\left(s_{k}\right) b \in \operatorname{nil}(R)$. Consider the generalized power series $f b=f C_{b}^{0} \in\left[\left[R^{S, \leq}\right]\right]$. Clearly, $N(f b)$ is generated by $f\left(s_{1}\right) b, f\left(s_{2}\right) b, \ldots, f\left(s_{n}\right) b$, where $s_{i} \leq s_{j}$ if $i \leq j$, and $f\left(s_{i}\right) b \notin \operatorname{nil}(R)$ implies $f b \notin n i l\left(\left[\left[R^{S, \leq}\right]\right]\right)$. It is easy to see that $f b$ has weak degree at most $k-1$. By the minimality of $k$, we know that there exists $c \in R$ with $f \cdot b \cdot c=f \cdot(b c)$ weak good. But this contradicts the fact that $f$ is a counterexample to the statement.

Proposition 3.7. Let $R$ be a semicommutative right Noetherian ring, $(S$, $\leq)$ a strictly totally ordered monoid. Then $N$ Ass $\left(\left[\left[R^{S, \leq}\right]\right]\right)=\left\{\left[\left[\wp^{S, \leq}\right]\right] \mid \wp \in\right.$ $N A s s(R)\}$.

Proof. We first prove $\supseteq$. Let $\wp \in N A s s(R)$. By definition, there exists a right ideal $I \nsubseteq \operatorname{nil}(R)$ with $I$ an $R$-prime ideal and $\wp=N_{R}(I)$. It suffices to prove

$$
\left[\left[\wp^{S, \leq}\right]\right]=N_{\left[\left[R^{S, \leq}\right]\right]}\left(\left[\left[I^{S, \leq}\right]\right]\right),
$$

and

$$
\left[\left[I^{S, \leq}\right]\right] \text { is }\left[\left[R^{S, \leq}\right]\right] \text {-prime. }
$$

For Eq.(1), let $f \in\left[\left[I^{S, \leq}\right]\right]$ and let $g \in\left[\left[\wp^{S, \leq}\right]\right]$. Then for any $u, v \in S$, since $f(u) \in I$ and $g(v) \in \wp$, we obtain $f(u) g(v) \in \operatorname{nil}(R)$. Applying Corollary 2.8 yields that $f g \in \operatorname{nil}\left(\left[\left[R^{S, \leq}\right]\right]\right)$. Hence $\left[\left[\wp^{S, \leq}\right]\right] \subseteq N_{\left[\left[R^{S,} \leq\right]\right]}\left(\left[\left[I^{S, \leq}\right]\right]\right)$.

Conversely, if $g \in N_{\left[\left[R^{S, \leq}\right]\right]}\left(\left[\left[I^{S, \leq]}\right]\right)\right.$, then $f g \in \operatorname{nil}\left(\left[\left[R^{S, \leq]}\right]\right)\right.$ for all $f \in$ $\left[\left[I^{S, \leq}\right]\right]$. In particular, for any $b \in I, C_{b}^{0} g \in \operatorname{nil}\left(\left[\left[R^{S, \leq}\right]\right]\right)$. Thus, by Corollary 2.8, $b g(s) \in \operatorname{nil}(R)$ for any $s \in S$, and so $g(s) \in N_{R}(I)=\wp$ for any $s \in S$. Hence $g \in\left[\left[\wp^{S, \leq}\right]\right]$, and so $N_{\left[\left[R^{S, \leq}\right]\right]}\left(\left[\left[I^{S, \leq}\right]\right]\right) \subseteq\left[\left[\wp^{S, \leq}\right]\right]$. Therefore $\left[\left[\wp^{S, \leq}\right]\right]=$ $N_{\left[\left[R^{S,} \leq\right]\right]}\left(\left[\left[I^{S, \leq}\right]\right]\right)$.

Note that the right ideal $I$ is an $R$-prime ideal. Then we have $I \nsubseteq \operatorname{nil}(R)$. Thus

$$
\left[\left[I^{S, \leq}\right]\right] \not\left[\left[\operatorname{nil}(R)^{S, \leq}\right]\right]=\operatorname{nil}\left(\left[\left[R^{S, \leq}\right]\right]\right) .
$$

To see (2), we must show that if a right ideal $\mho \nsubseteq \operatorname{nil}\left(\left[\left[R^{S, \leq}\right]\right]\right)$ and $\mho \subseteq\left[\left[I^{S, \leq}\right]\right]$, then

$$
N_{\left[\left[R^{S, \leq}\right]\right]}(\mho)=N_{\left[\left[R^{S, \leq}\right]\right]}\left(\left[\left[I^{S, \leq}\right]\right]\right) .
$$


To this end, let $C_{\mho}=\bigcup_{f \in \mho} C_{f}$, where $C_{f}=\{f(s) \mid s \in S\}$, and let $\wp_{0}$ denote the right ideal of $R$ generated by $C_{\mho}$. Since $\mho \nsubseteq \operatorname{nil}\left(\left[\left[R^{S, \leq}\right]\right]\right)=\left[\left[\operatorname{nil}(R)^{S, \leq}\right]\right]$, $C_{\mho} \nsubseteq \operatorname{nil}(R)$, and hence $\wp_{0} \subseteq I, \wp_{0} \nsubseteq \operatorname{nil}(R)$. So we have $N_{R}\left(\wp_{0}\right)=N_{R}(I)=\wp$ because $I$ is $R$-prime. Since $N_{\left[\left[R^{S, \leq]]}\right.\right.}(\mho) \supseteq N_{\left[\left[R^{S, \leq]]}\right.\right.}\left(\left[\left[I^{S, \leq]]}\right)\right.\right.$ is clear, it suffices to show that

$$
N_{\left[\left[R^{S, \leq]]}\right.\right.}(\mho) \subseteq N_{\left[\left[R^{S, \leq]]}\right.\right.}\left(\left[\left[I^{S, \leq}\right]\right]\right) .
$$

We now assume that $g \in N_{\left[\left[R^{S,} \leq\right]\right]}(\mho)$, then $f g \in \operatorname{nil}\left(\left[\left[R^{S, \leq]]}\right)\right.\right.$ for every $f \in \mho$. By Corollary 2.8, we obtain $f(u) g(v) \in \operatorname{nil}(R)$ for all $u, v \in S$. It follows from Lemma 2.4 that $f(u) R g(v) \subseteq \operatorname{nil}(R)$ for all $u, v \in S$. Thus $g(v) \in N_{R}\left(\wp_{0}\right)=$ $N_{R}(I)=\wp$ for all $v \in S$, and so $g \in\left[\left[\wp^{S, \leq]}\right]=N_{\left[\left[R^{S, \leq}\right]\right]}\left(\left[\left[I^{S, \leq}\right]\right]\right)\right.$. Hence $N_{\left[\left[R^{S, \leq]]}\right.\right.}(\mho) \subseteq N_{\left[\left[R^{S,} \leq\right]\right]}\left(\left[\left[I^{S, \leq]]}\right)\right.\right.$ is proved, and so is $\supseteq$ in Proposition 3.7.

Now we turn our attention to proving $\subseteq$ in Proposition 3.7. Let $I \in$ $N A s s\left(\left[\left[R^{S, \leq}\right]\right]\right)$. By definition, we have an $\left[\left[R^{S, \leq}\right]\right]$-prime ideal $£$ with $I=$ $N_{\left[\left[R^{S, \leq]]}\right.\right.}(£)$. Pick any $\pi \in £$, and $\pi \notin \operatorname{nil}\left(\left[\left[R^{S, \leq}\right]\right]\right)$. By $\pi \notin \operatorname{nil}\left(\left[\left[R^{S, \leq}\right]\right]\right)$ and Lemma 3.6, we may assume that $\pi$ is a weak good generalized power series, and $N \operatorname{deg}(\pi)=k$. Set $£_{0}=\pi\left[\left[R^{S, \leq}\right]\right]$, which is a principally right ideal generated by $\pi$. Note that $\pi \notin \operatorname{nil}\left(\left[\left[R^{S, \leq]]}\right)\right.\right.$, so we get

$$
£_{0}=\pi\left[\left[R^{S, \leq}\right]\right] \nsubseteq\left[\left[\operatorname{nil}(R)^{S, \leq}\right]\right]=\operatorname{nil}\left(\left[\left[R^{S, \leq}\right]\right]\right) .
$$

Then we have

$$
N_{\left[\left[R^{S, \leq}\right]\right]}(£)=N_{\left[\left[R^{S, \leq]]}\right.\right.}\left(£_{0}\right)=N_{\left[\left[R^{S, \leq]]}\right.\right.}\left(\pi \left[\left[R^{S, \leq]]}\right)=I,\right.\right.
$$

because $£$ is $\left[\left[R^{S, \leq}\right]\right.$-prime. Let $C_{\pi}=\{\pi(s) \mid s \in S\}$, and let $N(\pi)$ be the right ideal of $R$ generated by $C_{\pi}$. Since $R$ is a right Noetherian ring, we can find

$$
s_{1}<s_{2}<\cdots<s_{n}
$$

such that $N(\pi)$ is generated by $n$ elements

$$
\pi\left(s_{1}\right), \pi\left(s_{2}\right), \ldots, \pi\left(s_{n}\right) .
$$

Since $N \operatorname{deg}(\pi)=k$, we have $\pi\left(s_{k}\right) \notin n i l(R)$, and $N_{R}\left(\pi\left(s_{k}\right)\right) \subseteq N_{R}\left(\pi\left(s_{i}\right)\right)$ if $i \leq k$, and $\pi\left(s_{i}\right) \in \operatorname{nil}(R)$ if $i>k$. Considering the right ideal $\pi\left(s_{k}\right) R$, and assuming that $U=N_{R}\left(\pi\left(s_{k}\right) R\right)$, we wish to claim that $I=\left[\left[U^{S, \leq}\right]\right]$. Let $\alpha \in\left[\left[U^{S, \leq}\right]\right]$. Then for each $v \in S, \alpha(v) \in U=N_{R}\left(\pi\left(s_{k}\right) R\right)$, and so $\pi\left(s_{k}\right) R \alpha(v) \subseteq \operatorname{nil}(R)$. Since $\pi$ is a weak good generalized power series, and $N \operatorname{deg}(\pi)=k$, we have

$$
\pi\left(s_{i}\right) R \alpha(v) \subseteq \operatorname{nil}(R) \quad \text { for all } 1 \leq i \leq k .
$$

On the other hand, for all $i>k, \pi\left(s_{i}\right) \in \operatorname{nil}(R)$, thus we have

$$
\pi\left(s_{i}\right) R \alpha(v) \subseteq \operatorname{nil}(R) \quad \text { for all } 1 \leq i \leq n .
$$

Since $N(\pi)$ is generated by $\pi\left(s_{i}\right), 1 \leq i \leq n$, for each $u \in S$, there exist $r_{i} \in R$, $1 \leq i \leq n$, such that

$$
\pi(u)=\pi\left(s_{1}\right) r_{1}+\pi\left(s_{2}\right) r_{2}+\cdots+\pi\left(s_{n}\right) r_{n} .
$$


Thus we obtain

$$
\pi(u) R \alpha(v)=\left(\sum_{i=1}^{n} \pi\left(s_{i}\right) r_{i}\right) R \alpha(v) \subseteq \operatorname{nil}(R) .
$$

Hence for any $h \in\left[\left[R^{S, \leq}\right]\right]$ and any $u, w, v \in S$, we have $\pi(u) h(w) \alpha(v) \in$ $\operatorname{nil}(R)$, and so by Corollary 2.8, we have $\pi h \alpha \in \operatorname{nil}\left(\left[\left[R^{S, \leq}\right]\right]\right)$. Thus $\alpha \in$ $N_{\left[\left[R^{S,} \leq\right]\right]}\left(\pi\left[\left[R^{S, \leq]]}\right)=I\right.\right.$. Hence $\left[\left[U^{S,} \leq\right]\right] \subseteq I$.

Conversely, let

$$
\beta \in I=N_{\left[\left[R^{S, \leq]]}\right.\right.}(£)=N_{\left[\left[R^{S, \leq}\right]\right]}\left(\pi\left[\left[R^{S, \leq}\right]\right]\right) .
$$

Then for any $C_{r}^{0} \in\left[\left[R^{S, \leq}\right]\right]$, we have $\pi C_{r}^{0} \beta \in \operatorname{nil}\left(\left[\left[R^{S, \leq}\right]\right]\right)$. Then by Corollary 2.8, we get $\pi\left(s_{k}\right) r \beta(v) \in \operatorname{nil}(R)$ for all $r \in R$ and $v \in S$. Hence $\beta(v) \in$ $N_{R}\left(\pi\left(s_{k}\right) R\right)=U$ for each $v \in S$, and so $\beta \in\left[\left[U^{S, \leq}\right]\right]$. Hence $I \subseteq\left[\left[U^{S, \leq}\right]\right]$. Therefore $I=\left[\left[U^{S,} \leq\right]\right]$.

We are now to check that the principally right ideal $\pi\left(s_{k}\right) R$ is $R$-prime. Since $\pi\left(s_{k}\right) \notin \operatorname{nil}(R), \pi\left(s_{k}\right) R \nsubseteq \operatorname{nil}(R)$. Assume that a right ideal $Q \subseteq \pi\left(s_{k}\right) R$, and $Q \nsubseteq \operatorname{nil}(R)$. Then $N_{R}(Q) \supseteq N_{R}\left(\pi\left(s_{k}\right) R\right)$ is clear. Now we show that

$$
N_{R}(Q) \subseteq N_{R}\left(\pi\left(s_{k}\right) R\right) .
$$

Set $W=\left\{\pi r=\pi C_{r}^{0} \mid r \in Q\right\}$, and let $W\left[\left[R^{S, \leq}\right]\right]$ be the right ideal of $\left[\left[R^{S, \leq}\right]\right]$ generated by $W$. It is obvious that $W\left[\left[R^{S, \leq}\right]\right] \subseteq \pi\left(\left[\left[R^{S, \leq}\right]\right]\right)$. Since $Q \nsubseteq \operatorname{nil}(R)$, there exists $a \in R$ such that $\pi\left(s_{k}\right) a \in Q$ and $\pi\left(s_{k}\right) a \notin n i l(R)$. If

$$
\left(\pi \cdot \pi\left(s_{k}\right) a\right)\left(s_{k}\right)=\left(\pi C_{\left(\pi\left(s_{k}\right) a\right)}^{0}\right)\left(s_{k}\right)=\pi\left(s_{k}\right) \pi\left(s_{k}\right) a \in \operatorname{nil}(R),
$$

then we have

$$
\pi\left(s_{k}\right) \pi\left(s_{k}\right) a \in \operatorname{nil}(R) \Rightarrow \pi\left(s_{k}\right) a \pi\left(s_{k}\right) \in \operatorname{nil}(R) \Rightarrow \pi\left(s_{k}\right) a \in \operatorname{nil}(R) .
$$

This contradicts to the fact that $\pi\left(s_{k}\right) a \notin n i l(R)$. Thus $\left(\pi \cdot \pi\left(s_{k}\right) a\right)\left(s_{k}\right) \notin$ $\operatorname{nil}(R)$, and so by Corollary 2.6,

$$
\pi \cdot \pi\left(s_{k}\right) a \notin \operatorname{nil}\left(\left[\left[R^{S, \leq}\right]\right]\right) .
$$

This implies that $W\left[\left[R^{S, \leq}\right]\right] \nsubseteq \operatorname{nil}\left(\left[\left[R^{S, \leq}\right]\right]\right)$. Since $£$ is $\left[\left[R^{S, \leq}\right]\right]$-prime, we obtain

$$
N_{\left[\left[R^{S, \leq}\right]\right]}\left(W\left[\left[R^{S, \leq}\right]\right]\right)=N_{\left[\left[R^{S,} \leq\right]\right]}\left(\pi\left[\left[R^{S, \leq}\right]\right]\right)=I=\left[\left[U^{S, \leq}\right]\right] .
$$

Suppose $q \in N_{R}(Q)$. Then $r q \in \operatorname{nil}(R)$ for each $r \in Q$. Then for any $r \in Q$, and any $\pi r \alpha=\pi C_{r}^{0} \alpha \in W\left[\left[R^{S, \leq}\right]\right]$ and any $s \in S$,

$$
\left(\pi C_{r}^{0} \alpha C_{q}^{0}\right)(s)=\sum_{(u, v) \in X_{s}(\pi, \alpha)} \pi(u) r \alpha(v) q .
$$

From $r q \in \operatorname{nil}(R)$ and Lemma 2.4, we obtain $\pi(u) r \alpha(v) q \in \operatorname{nil}(R)$ for any $u, v \in S$, and so $\left(\pi C_{r}^{0} \alpha C_{q}^{0}\right)(s) \in \operatorname{nil}(R)$. Thus by Corollary 2.6, $\pi C_{r}^{0} \alpha C_{q}^{0} \in$ $\operatorname{nil}\left(\left[\left[R^{S, \leq}\right]\right]\right)$. Hence

$$
C_{q}^{0} \in N_{\left[\left[R^{S,}\right]\right]}\left(W\left(\left[\left[R^{S, \leq}\right]\right]\right)\right)=I=\left[\left[U^{S, \leq}\right]\right],
$$


and so $q \in U=N_{R}\left(\pi\left(s_{k}\right) R\right)$. Hence $N_{R}(Q) \subseteq N_{R}\left(\pi\left(s_{k}\right) R\right)$, and this implies that $N_{R}(Q)=N_{R}\left(\pi\left(s_{k}\right) R\right)$. Thus we obtain $\pi\left(s_{k}\right) R$ is $R$-prime.

Assembling the above results, we finish the proof of Proposition 3.7.

Corollary 3.8. Let $R$ be a semicommutative Noetherian ring. Then

$$
N A s s(R[[x]])=\{\wp[[x]] \mid \wp \in N A s s(R)\} .
$$

Acknowledgements. The authors are deeply indebted to the referee for many helpful comments and suggestions for the improvement of this paper.

\section{References}

[1] D. D. Anderson and V. Camillo, Armendariz rings and Gaussian rings, Comm. Algebra 26 (1998), no. 7, 2265-2272.

[2] S. Annin, Associated primes over skew polynomial rings, Comm. Algebra 30 (2002), no. $5,2511-2528$

[3] - Associated primes over Ore extension rings, J. Algebra Appl. 3 (2004), no. 2, $193-205$.

[4] J. A. Beachy and W. D. Blair, Rings whose faithful left ideals are cofaithful, Pacific J. Math. 58 (1975), no. 1, 1-13.

[5] J. Brewer and W. Heinzer, Associated primes of principal ideals, Duke Math. J. 41 (1974), 1-7.

[6] G. A. Elliott and P. Ribenboim, Fields of generalized power series, Arch. Math. (Basel) 54 (1990), no. 4, 365-371.

[7] C. Faith, Associated primes in commutative polynomial rings, Comm. Algebra 28 (2000), no. 8, 3983-3986.

[8] Y. Hirano, On annihilator ideals of a polynomial ring over a noncommutative ring, J. Pure Appl. Algebra 168 (2002), no. 1, 45-52.

[9] C. Y. Hong, N. K. Kim, T. K. Kwak, and Y. Lee, Extensions of zip rings, J. Pure Appl. Algebra 195 (2005), no. 3, 231-242.

[10] J. Lambek, On the representation of modules by sheaves of factor modules, Canad. Math. Bull. 14 (1971), 359-368.

[11] Z. Liu, PF-rings of generalised power series, Bull. Austral. Math. Soc. 57 (1998), no. $3,427-432$.

[12] _ Injectivity of modules of generalized inverse polynomials, Comm. Algebra 29 (2001), no. 2, 583-592.

[13] _ Special properties of rings of generalized power series, Comm. Algebra 32 (2004), no. 8, 3215-3226.

[14] G. Marks, On 2-primal Ore extensions, Comm. Algebra 29 (2001), no. 5, 2113-2123.

[15] L. Ouyang, Ore extensions of weak zip rings, Glasg. Math. J. 51 (2009), no. 3, 525-537.

[16] L. Ouyang and Y. Chen, On weak symmetric rings, Comm. Algebra 38 (2010), no. 2, 697-713.

[17] P. Ribenboim, Rings of generalized power series: Nilpotent elements, Abh. Math. Sem. Univ. Hamburg 61 (1991), 15-33.

[18] _ Noetherian rings of generalized power series, J. Pure. Appl. Algebra 79 (1992), no. 3, 293-312

[19] Semisimple rings and von Neumann regular rings of generalized power series, J. Algebra 198 (1997), no. 2, 327-338.

[20] R. C. Shock, Polynomial rings over finite dimensional rings, Pacific J. Math. 42 (1972), $251-257$. 
Department of Mathematics

Hunan University of Science and Technology

Xiangtan, Hunan 411201, P. R. China

E-mail address: Ouyanglqtxy@163.com 